\title{
Effects of solutes on dislocation nucleation from grain boundaries
}

\author{
Valery Borovikov ${ }^{1 \pm}$, Mikhail I. Mendelev ${ }^{1}$ and Alexander H. King ${ }^{1,2}$
}

\begin{abstract}
When grain sizes are reduced to the nanoscale, grain boundaries (GB) become the dominant sources of the dislocations that enable plastic deformation. We present the first molecular dynamics (MD) study of the effect of substitutional solutes on the dislocation nucleation process from GBs during uniaxial tensile deformation. A simple bi-crystal geometry is utilized in which the nucleation and propagation of dislocations away from a GB is the only active mechanism of plastic deformation. Solutes with atomic radii both larger and smaller than the solvent atomic radius were considered. Although the segregation sites are different for the two cases, both produce increases in the stress required to nucleate a dislocation. MD simulations at room temperature revealed that this increase in the nucleation stress is associated with changes of the GB structure at the emission site caused by dislocation emission, leading to increases in the heats of segregation of the solute atoms, which cannot diffuse to lower-energy sites on the timescale of the nucleation event. These results contribute directly to understanding the strength of nanocrystalline materials, and suggest suitable directions for nanocrystalline alloy design leading toward structural applications.
\end{abstract}




\section{Introduction}

Nanocrystalline metals have been the subject of numerous studies because of their potential use in high strength applications (Chowdhury et al., 2015; Chowdhury et al., 2016; Min et al., 2014; Salehinia et al., 2014; Yuan et al., 2016). The large densities of grain boundaries in these materials dramatically change the deformation behavior from that observed in their conventional polycrystalline counterparts (Burbery et al., 2015; Hahn and Meyers, 2015; Ivanisenko et al., 2016; Khalajhedayati et al., 2016; Upadhyay et al., 2016; Zhang et al., 2015). Rapid grain growth and the consequent loss of their superior mechanical properties limit the application of these remarkable materials, however, so solute additions are commonly used to stabilize them against microstructural coarsening (Frolov et al., 2012; Gottstein and Shvindlerman, 1999; Millett et al., 2007). Solutes segregated at grain boundaries (GB) for the purpose of microstructural stabilization may also significantly affect plastic deformation mechanisms such as grain boundary sliding, grain rotation, twinning, dislocation slip, etc (Kuhr et al., 2016; Schaefer et al., 2011; Tschopp et al., 2014; Vo et al., 2011). The mechanisms of these effects have been the subjects of recent molecular dynamics (MD) simulation studies. For example, a strong influence of solute segregation at GBs on the strength of dilute nanocrystalline $\mathrm{Cu}$ alloys was predicted in (Vo et al., 2011) and later confirmed experimentally in (Ozerinc et al., 2012). Solute atoms with a large size mismatch with the $\mathrm{Cu}$ host were shown to be the most effective. In (Rajgarhia et al., 2010), the effect of Sb solute atoms on the tensile deformation behavior of nanocrystalline $\mathrm{Cu}$ was modeled. It was observed that the yield stress increases with increasing concentrations of $\mathrm{Sb}$ atoms at the GBs. However, solute equilibration was not achieved prior to deformation in this study, as the solute atoms were introduced simply by randomly removing $\mathrm{Cu}$ atoms at the GBs and replacing them with $\mathrm{Sb}$. In contrast with all of the above cases, the results presented in (Jang et al., 2008) indicate a decrease in the yield stress of nanocrystalline $\mathrm{Al}$ due to the addition of $\mathrm{Pb}$ solute atoms at the GBs. They also observed that $\mathrm{Pb}$ segregation at GBs in nanocrystalline Al leads to the suppression of partial dislocation nucleation during straining.

In all of the cases cited above, large, 3D nano-polycrystalline models were used to provide a semblance of the complexity of real materials, but it was consequently difficult to isolate any specific mechanisms governing the plastic deformation process. Moreover, the very small grain sizes in the MD simulation cells may have biased the plastic deformation mechanism in favor of grain boundary sliding and/or grain rotation, so the effect of solutes on dislocation nucleation has therefore not yet been studied systematically. In this paper we report the results of MD simulations using a simple bicrystal geometry for which the nucleation and propagation of dislocations from a GB is the only mechanism of the plastic deformation.

In the present work, we have simulated the nucleation of dislocations from $\Sigma 11(332)[110]$ symmetric tilt grain boundaries (STGBs). The <110> rotation axis dominates the grain boundary character distributions of face-centered cubic (fcc) materials (Randle, 2006) and this family includes a subset of interfaces which contain E structural units (Rittner and Seidman, 1996). These STGBs are able to emit dislocations at lower applied tensile stresses than grain boundaries that lack E units (Spearot et al., 2007; Tschopp et al., 2007) and because they operate as sources at lower stresses than other boundaries, they may determine the bulk yield stress. As model systems of potential 
practical interest, we have chosen $\mathrm{Cu}$ and $\mathrm{Ag}$ with a small addition of $\mathrm{Ag}$ and $\mathrm{Cu}$, respectively. While both pure $\mathrm{Cu}$ and $\mathrm{Ag}$ are fcc metals, they have very low mutual solubility (Massalski et al., 1990) so the solute is expected to segregate to the GBs in either host. Since $\mathrm{Ag}$ atoms are considerably larger than $\mathrm{Cu}$ atoms, the model also allows us to study the effects of solutes that are smaller than the host atoms, and vice versa.

The rest of the paper is organized as follows. In Section 2, we describe the methods used for MD simulations of plastic deformation in bi-crystals. The MD simulations demonstrate that the yield stress depends very strongly on the solute concentration, for both the positive and negative misfit cases. In Section 3, we develop an intuitive, thermodynamically-based hypothesis to explain the trends observed in the MD simulations. Finally we demonstrate that while it is successful within certain limits, this does not explain all of the details, and a more sophisticated theory is accordingly needed. We describe some of the features that should be included in order to provide greater fidelity.

\section{Molecular dynamics simulation}

Atomic interactions were described by an embedded-atom method (EAM) potential introduced in (Williams et al., 2006) and molecular dynamics simulations were carried out using the LAMMPS package (Plimpton, 1995). The simulation cells had periodic boundary conditions in all directions and contained two identical GBs normal to the z-direction (see Fig. 1a) and separated by $\sim 17 \mathrm{~nm}$. First, we determined the minimum-energy $\mathrm{GB}$ structures for pure $\mathrm{Cu}$, and pure $\mathrm{Ag}$ following the procedure described in (Duparc et al., 2005; Mishin and Farkas, 1998). The equilibrated GBs have structures with "zigzag" glide mirror planes containing type E structural units (see Fig. 1b) and provide several distinct segregation sites. In both of the pure materials, the atoms at sites $\mathrm{A}^{\prime}$ and $\mathrm{D}^{\prime}$ are in a state of predominantly hydrostatic internal stress, in which the absolute values of the off-diagonal components of the local stress tensor are smaller than the diagonal ones. Site $\mathrm{A}^{\prime}$ experiences a large tensile hydrostatic stress, and is therefore the most favorable site for segregation of the $\mathrm{Ag}$ atom in $\mathrm{Cu}$, since $\mathrm{Ag}$ is larger than the $\mathrm{Cu}$ that it replaces. Site $\mathrm{D}^{\prime}$ is strongly under compression and is thus the most favorable site for segregation of the relatively undersized $\mathrm{Cu}$ atom in $\mathrm{Ag}$. This simple rationale for site selection is, of course, only reliable at very low solute concentrations for which a solute atom segregated on the GB has a very low probability of having another solute atom as a neighbor, because a solute atom on one site can considerably change the segregation energy for the nearby sites.

To simulate the effect of solutes on the dislocation nucleation, we first prepared smaller simulation cells in the same manner as described above. The simulation cells had periodic boundary conditions in all directions and contained only solvent atoms. The cell was longer in the $\mathrm{z}$-direction (normal to the grain boundaries) and relatively small in the $\mathrm{x}$ - and y-directions $\left(2.4 \times 2.3 \times 33.6 \mathrm{~nm}^{3}\right.$ for $\mathrm{Cu}$ and $2.7 \times 2.6 \times 38.0 \mathrm{~nm}^{3}$ for $\left.\mathrm{Ag}\right)$. It contained 15660 atoms and enclosed two identical GBs normal to the z-direction. In spite of the small simulation sizes in the $\mathrm{x}$ - and $\mathrm{y}$-directions, the cell was large enough to include several repeats of the periodic GB structure.

For each doping level, the chosen number of solvent atoms was replaced by solute atoms in a special swap region near the GBs (see Fig. 1a) that extended approximately 3 $\mathrm{nm}$ in the direction normal to the GB. Monte Carlo (MC) relaxation was performed on 
the rigid lattice within the first swap region, followed by the same MC relaxation for the second swap region. As expected, the solutes exhibited a strong tendency to segregate to the $\mathrm{GBs}$ for both $\mathrm{Ag}$ in $\mathrm{Cu}$ and $\mathrm{Cu}$ in $\mathrm{Ag}$, justifying the use of a localized swap region and reducing the number of $\mathrm{MC}$ trials needed to reach an equilibrium solute distribution. The energy of the system quickly dropped during each MC simulation, as can be seen in Fig. 2.

Next we performed short MD simulations to initially equilibrate the model at $\mathrm{T}=300 \mathrm{~K}$ and zero applied stress. During this run the system energy drops considerably, again, because the $\mathrm{Cu}-\mathrm{Ag}$ equilibrium separation is different than $\mathrm{Cu}-\mathrm{Cu}$ equilibrium separation. Finally, the models were subjected to a hybrid MC/MD relaxation (Sadigh et al., 2012). In this technique, the MD simulations were interrupted at every 20th MD step to perform one MC trial exchange event (swapping a solute atom with a matrix atom within the same swap region). On average, each solute atom was subjected to 2500 trial exchanges. Figure 2 shows that the energy decreases for approximately 2,000,000 MD steps and then reaches an equilibrium value. The MC/MD equilibrated model was then translated and repeated 7 times in the $\mathrm{x}$ - and $\mathrm{y}$-directions so the final model contained 767340 atoms.

The hybrid MC/MD simulations revealed that for the case of small Ag concentrations in $\mathrm{Cu}$, the $\mathrm{Ag}$ solute atoms segregate exclusively to the $\mathrm{A}^{\prime}$ sites. When all of the sites $\mathrm{A}^{\prime}$ are filled, segregation to the $\mathrm{B}_{3}^{\prime}$ occurs, and when most of the $\mathrm{B}_{3}^{\prime}$ sites are occupied, segregation into other sites $\left(B_{1}^{\prime}, C^{\prime}\right)$ starts to occur. For the case of $\mathrm{Cu}$ solutes in $\mathrm{Ag}$, only the sites $\mathrm{D}^{\prime}$ are occupied at low $\mathrm{Cu}$ concentrations. When the most of the $\mathrm{D}^{\prime}$ sites are occupied, segregation into the $\mathrm{C}^{\prime}$ sites is observed. The presence of $\mathrm{Cu}$ atoms in the $\mathrm{D}^{\prime}$ and $\mathrm{C}^{\prime}$ sites influences the segregation energies of the sites nearby, so as the solute concentration increases further, segregation into the $\mathrm{B}_{2}^{\prime}$ sites is also observed. Finally, at the highest concentrations considered in the present study, segregation into the $\mathrm{B}_{4}^{\prime}$ sites and a couple of non-principal segregation sites was also observed.

Prior to tensile loading, each simulation cell was annealed at $T=500 \mathrm{~K}$ and $\sigma_{\alpha \alpha}=0 \quad(\alpha=x, y$ or $z)$ for $0.2 \mathrm{~ns}$, cooled down to $T=300 \mathrm{~K}$ and equilibrated at this temperature at $\sigma_{\alpha \alpha}=0$. Uniaxial tensile loading simulations were carried out with a constant engineering strain rate of $10^{8} \mathrm{~s}^{-1}$ applied in the direction normal to the GB plane, while the stresses in the other two directions were held at zero. During the tensile loading $\sigma_{\mathrm{zz}}$ initially increases with applied strain, reaches a maximum value, corresponding to the emission of the first dislocation, and then drops substantially (see Fig. 3). Because $\mathrm{Cu}$ and $\mathrm{Ag}$ have rather small stacking fault energies, we only observed the emission of $60^{\circ}$ partial dislocations in these simulations (see the discussion in (Borovikov et al., 2015)). The peak stress value associated with the first dislocation emission was treated as the effective yield stress in this work.

The dependence of the yield stress on solute concentration is shown in Fig. 4. In both cases, for relatively small solute concentrations, segregation at the GB leads to a significant increase in the tensile strength. When the solute atoms substitute approximately $30 \%$ of the solvent atoms at the GB, the strengthening effect levels off.

\section{Discussion}


Our results address the nucleation of the first dislocation from a specific grain boundary, which is important if that event always occurs at a lower stress than any other available deformation process. The emission of subsequent dislocations, the emission of dislocations from other grain boundaries, the operation of other sources at surfaces and triple junctions all affect yield, and the interactions of dislocations with other grain boundaries, and with each other in the grain interiors, contribute to work hardening: each of these processes may also be affected by solute atoms in ways that remain to be evaluated.

The MD simulations performed in the present study demonstrate that addition of solutes can dramatically affect the nucleation of dislocations from GBs, leading to considerable increases in the yield stress. It should be noted, here, that the strain rate used in the MD simulations is much higher than that typically used in experiments. No solute diffusion occurs at $\mathrm{T}=300 \mathrm{~K}$ during the MD simulations while some short range diffusion is possible under typical experimental conditions (e.g., see the consideration of a similar phenomenon in (Curtin et al., 2006)). This local diffusion may partly suppress the effects observed here (Schafer et al., 2013). Since the goal of the present study was just to demonstrate the possible effect of solutes on the dislocation nucleation from GBs we did not attempt to combine the MD simulation of the tensile loading with any method to incorporate the solute diffusion. Instead below we focus on rationalizing our simulation results.

To understand why solutes affect dislocation nucleation, we start from the analysis of nucleation from the same GB in pure metals presented in (Spearot, 2008; Tschopp et al., 2007; Tucker et al., 2010). As a result of dislocation emission, the part of the crystal, containing site $\mathrm{A}^{\prime}$, shifts towards the $\mathrm{E}$ structural unit, while the part containing sites $\mathrm{C}^{\prime}, \mathrm{D}^{\prime}$ and $\mathrm{B}^{\prime}{ }_{4}$, shifts in the opposite direction (see Fig. 1b). Since the solute atoms shift along with the solvent atoms, they may end up in the positions that are not as favorable for segregation as were their original sites. For example, the volume associated with the site $\mathrm{A}^{\prime}$ decreases considerably after a dislocation is emitted from the E unit (Spearot, 2008). Therefore, the new location of the solute atom may be less favorable for the relatively large $\mathrm{Ag}$ atom in $\mathrm{Cu}$ than it was before dislocation emission. To characterize this effect, consider two processes. In the first one, the dislocation is emitted from the GB in a pure metal. The change of the energy associated with the process is

$$
\Delta E_{d}^{p}=E_{d}^{p}-E^{p}
$$

where $E_{d}^{p}$ and $E^{p}$ are respectively the energies of the pure system with and without an emitted dislocation $\left(E_{d}^{p}\right.$ depends on how far the dislocation travelled from the GB which will discussed below). The second process is dislocation emission in the presence of solutes which can be characterized by the following quantity:

$$
\Delta E_{d}^{s}=E_{d}^{s}-E^{s}
$$

where $E_{d}^{s}$ and $E^{s}$ are energies of the system with solutes, with and without an emitted dislocation. The effect of the solutes can be then characterized as:

$$
\Delta E=\left(\Delta E_{d}^{s}-\Delta E_{d}^{p}\right) / N_{G B}^{s}
$$



dislocation is emitted (the system can have other solutes atoms). It is assumed that all energies in the RHS of both Eqs. (1) and (2) describe completely relaxed states and the dislocation is located at the same distance from the GB in every case. In practice, it is difficult to satisfy the latter condition because the dislocation will either keep propagating or come back to the GB location from which it was emitted, and annihilate during the minimization step. To overcome this problem we used the simulation cell shown in Fig. $1 \mathrm{c}$ where the dislocation has propagated all the way to the second GB (the top GB in the figure, which contains no solute atoms). This ensures that solute site reorganization near the dislocation-nucleating GB is the only effect in play.

If there is just one solute atom in the system, $\Delta E$ describes the change in the heat of segregation associated with emission of the dislocation from the $E$ unit where the solute atom segregates (we refer to this quantity as $\Delta E_{1}$ below). Table I shows that $\Delta E$ does not correlate with the heat of segregation itself; there are no correlations even between signs of these quantities. However, $\Delta E_{1}$ indeed increases for the most favorable initial segregation sites, once the dislocation is emitted from the GB. One might conclude that the simple model represented by Eq. (3) can explain the effect of segregated solutes on the value of the yield stress. The actual mechanism, however, is rather more complicated.

Table I. Heats of segregation and effects of solutes on the change in energy associated with the dislocation emission. All energies are expressed in eV/atom. The values for the most favorable segregation sites are shown in bold. The values of $\Delta E_{3}$ are shown in italic if the GB experiences a reconstruction during the tensile loading simulation.

\begin{tabular}{|c|c|c|c|c|c|c|c|c|}
\hline \multirow{2}{*}{ Site } & \multicolumn{4}{|c|}{$\mathrm{Ag}$ in $\mathrm{Cu}$} & \multicolumn{4}{c|}{$\mathrm{Cu}$ in $\mathrm{Ag}$} \\
\cline { 2 - 10 } & $E_{\text {seg }}$ & $\Delta E_{1}$ & $\Delta E_{3}$ & $\Delta E_{3}^{1 \%}$ & $E_{\text {seg }}$ & $\Delta E_{1}$ & $\Delta E_{3}$ & $\Delta E_{3}^{1 \%}$ \\
\hline $\mathrm{A}^{\prime}$ & $\mathbf{- 0 . 4 2 0}$ & $\mathbf{0 . 1 8 2}$ & $\mathbf{0 . 3 0 6}$ & $\mathbf{0 . 3 3 9}$ & 0.130 & -0.045 & -0.019 & -0.023 \\
\hline $\mathrm{B}_{1}^{\prime}$ & -0.210 & 0.205 & 0.090 & 0.118 & -0.050 & -0.042 & 0.017 & 0.015 \\
\hline $\mathrm{B}_{2}^{\prime}$ & -0.120 & 0.250 & 0.382 & 0.355 & 0.012 & -0.099 & -0.080 & -0.054 \\
\hline $\mathrm{B}_{3}^{\prime}$ & -0.170 & 0.107 & 0.308 & 0.523 & 0.050 & -0.049 & -0.004 & -0.029 \\
\hline $\mathrm{B}_{4}^{\prime}$ & -0.003 & -0.076 & -0.228 & -0.282 & -0.080 & 0.088 & 0.124 & 0.159 \\
\hline $\mathrm{C}^{\prime}$ & -0.010 & -0.208 & 0.284 & 0.492 & -0.015 & 0.081 & -0.025 & -0.055 \\
\hline $\mathrm{D}^{\prime}$ & 0.110 & -0.222 & 0.187 & 0.346 & $\mathbf{- 0 . 2 0 0}$ & $\mathbf{0 . 1 5 4}$ & $\mathbf{0 . 0 2 2}$ & $\mathbf{0 . 0 0 6}$ \\
\hline
\end{tabular}


First, we note that this simple model should be equally applicable to solute segregation at any GB site provided that there is no GB reconstruction during plastic deformation. The values of $\Delta E_{1}$ presented in Table I suggest that even if the solute segregates to a less favorable site, it can still dramatically increase the yield stress (e.g., Ag segregating into the $\mathrm{B}_{2}^{\prime}$ sites in $\mathrm{Cu}$ ). On the other hand, there are sites where the heat of segregation decreases after the emission of the dislocation (e.g., site $\mathrm{D}^{\prime}$ in the case of Ag solutes in $\mathrm{Cu}$ ). In this case, the addition of solutes should decrease the yield stress. To explore if there is a correlation between the value of $\Delta E_{1}$ and the yield stress we performed an additional series of MD simulations. This time we placed solute atoms only at every third equivalent segregation site along the $y$-axis in the simulation cell (an example is shown in Fig. 5). No MC/MD relaxation was carried out, but tensile deformation was performed exactly as before. Figure 6a shows that while some correlation still exists between the yield stress and the corresponding values of $\Delta E_{1}$ in the case of $\mathrm{Cu}$ solutes in $\mathrm{Ag}$, no correlation is observed in the case of Ag solutes in $\mathrm{Cu}$. Thus, the value of $\Delta E_{1}$ is not a reliable predictor of the yield stress.

The yield stresses shown in Fig. 6a were obtained using the models in which every third equivalent segregation site was occupied by a solute atom, but $\Delta E_{1}$ describes the change in energy when only one solute atom is present in the system and, therefore, it does not correctly account for the effects of closely-spaced solute atoms on each other. To evaluate these effects we determined the value of $\Delta E_{3}$ using Eq. (3) for the case where every third segregation site is occupied by a solute atom. Table I shows that the interaction effects are significant. For example, for the site $\mathrm{D}^{\prime}$ in the case of Ag solutes in $\mathrm{Cu}$, the value of $\Delta E$ changes from a large negative value to a large positive value, which explains why segregation at this site does not lead to a decrease in the yield stress. Figure $6 \mathrm{~b}$ shows the correlation between the yield stress and the value of $\Delta E_{3}$. In the case of $\mathrm{Cu}$ solutes in $\mathrm{Ag}$, the replacement of $\Delta E_{1}$ by $\Delta E_{3}$ did not result in a stronger correlation but for the case of $\mathrm{Ag}$ solutes in $\mathrm{Cu}$, the same replacement provided a much stronger correlation.

An additional caveat arises because the values of $\Delta E_{1}$ and $\Delta E_{3}$ were determined at zero deformation while a GB actually emits a dislocation only under applied strain. To test the effect of the deformation on the value of $\Delta E_{3}$ we determined $\Delta E_{3}^{1 \%}$ which is equivalent to $\Delta E_{3}$ except that all of the energies in Eqs. (1)-(2) where calculated using models deformed to a strain of $1 \%$. Figure $6 \mathrm{c}$ shows that accounting for the effect of deformation in this way leads to a slightly better correlation between the yield stress and the value of $\Delta E_{3}^{1 \%}$.

A simple model based on the interaction between the dislocation and the solutes segregated at GBs provides an intuitively satisfying explanation of the general trends observed in our MD simulations, but fails to properly explain the details. For example, based on the value of $\Delta E_{3}^{1 \%}$ for $\mathrm{Cu}$ segregated in the $\mathrm{D}^{\prime}$ site in $\mathrm{Ag}$ we should expect a very small effect on the yield stress, but the MD simulation demonstrates that it is large. This failure can be attributed to several factors that were not taken into account. First, the geometry used to determine $\Delta E_{3}^{1 \%}$ (Fig. 6c) does not correspond exactly to the geometry 
at the point of dislocation nucleation. The leading partial dislocation in Fig. 1c is far away from the GB from which it was emitted so that the interaction between the dislocation core and the solute atoms is much weaker than it was when the dislocation nucleated. Recall that the reason for using the configuration shown in Fig. 1c is that it is impossible to perform the energy minimization keeping the dislocation core at the specific distance from the GB associated with the point of nucleation. Fig. 1d vividly shows that the GB structure changes considerably at the moment when a dislocation nucleates: a fish-bone structure is formed consisting of dislocation embryos emanating from nearby sites and it is not obvious how to incorporate the interactions among these embryos into the model. Critically, the fish-bone structure does not form during the energy minimizations employed to determine all quantities in Eqs. (1)-(2), so there is reasonable doubt that these energies correspond exactly to the interactions of concern at the point of nucleation.

Finally we note that in real nanocrystalline materials, solutes will affect other mechanisms operating during plastic deformation. For example, solutes can affect the dislocation nucleation from free surfaces (e.g., see (Chen et al., 2015; Jennings et al., 2013; Zhu et al., 2008)), the GB sliding or grain rotation (e.g., see (Vo et al., 2011)). They can also affect post-nucleation dislocation motion (e.g., see (Olmsted et al., 2006; Olmsted et al., 2005)), and other effects are possible, too. These phenomena were not considered in the present study. Moreover, any real nanocrystalline material contains a set of different GBs and the solute effect of the dislocation nucleation should vary depending on the GB type. Thus, the overall solute effect on yield stress can be much more complicated than what is shown in Fig. 4. Because of the complicated interactions of different processes in polycrystalline materials, direct experimental validation of our results can only be provided using transmission electron microscopy or x-ray topography, ideally through in situ straining of controlled bicrystal specimens with misorientations and compositions corresponding to our simulations: it is now becoming possible to perform such experiments with local strain (hence stress) determination, and we look forward to the results from such efforts.

\section{Conclusions}

MC/MD simulations of the effect of solutes on dislocation nucleation from $\Sigma 11(332)[110]$ STGBs demonstrate that the addition of either oversized ( $\mathrm{Ag}$ in $\mathrm{Cu})$ or undersized $(\mathrm{Cu}$ in $\mathrm{Ag})$ solutes can dramatically increase the yield stress associated with this deformation mechanism. The increase is attributed to changes in the GB structure that occur during dislocation emission leading to increases in the heat of segregation, and thus the energy barrier for dislocation nucleation. However, this explanation is complicated by other factors that need to be accounted for to fully describe the nucleation process. While the present study has identified some of these factors, incorporating them in a theoretical model should be a subject for further research.

\section{Acknowledgements}

This work was supported by the U.S. Department of Energy (DOE), Office of Science, Basic Energy Sciences, Materials Science and Engineering Division. The research was performed at Ames Laboratory, which is operated for the U.S. DOE by Iowa State University under contract \# DE-AC02-07CH11358. 
Borovikov, V., Mendelev, M.I., King, A.H., LeSar, R., 2015. Effect of stacking fault energy on mechanism of plastic deformation in nanotwinned FCC metals. Modelling and Simulation in Materials Science and Engineering 23.

Burbery, N.J., Das, R., Ferguson, W.G., 2015. Modelling with variable atomic structure: Dislocation nucleation from symmetric tilt grain boundaries in aluminium. Computational Materials Science 101, 16-28. Chen, L.Y., He, M.R., Shin, J., Richter, G., Gianola, D.S., 2015. Measuring surface dislocation nucleation in defect-scarce nanostructures. Nature Materials 14, 707713.

Chowdhury, P., Sehitoglu, H., Abuzaid, W., Maier, H.J., 2015. Mechanical response of low stacking fault energy Co-Ni alloys - Continuum, mesoscopic and atomic level treatments. International Journal of Plasticity 71, 32-61.

Chowdhury, P., Sehitoglu, H., Maier, H.J., Rateick, R., 2016. Strength prediction in NiCo alloys - The role of composition and nanotwins. International Journal of Plasticity 79, 237-258.

Curtin, W.A., Olmsted, D.L., Hector, L.G., 2006. A predictive mechanism for dynamic strain ageing in aluminium-magnesium alloys. Nature Materials 5, 875-880.

Duparc, O.H., Larere, A., Lezzar, B., Khalfallah, O., Paidar, V., 2005. Comparison of the intergranular segregation for eight dilute binary metallic systems in the Sigma 11 ' $\{332\}$ tilt grain boundary. Journal of Materials Science 40, 3169-3176.

Frolov, T., Darling, K.A., Kecskes, L.J., Mishin, Y., 2012. Stabilization and strengthening of nanocrystalline copper by alloying with tantalum. Acta Materialia 60, 2158-2168.

Gottstein, G., Shvindlerman, L.S., 1999. Grain boundary migration in metals : thermodynamics, kinetics, applications. CRC Press, Boca Raton.

Hahn, E.N., Meyers, M.A., 2015. Grain-size dependent mechanical behavior of nanocrystalline metals. Materials Science and Engineering a-Structural Materials Properties Microstructure and Processing 646, 101-134. Honeycutt, J.D., Andersen, H.C., 1987. MOLECULAR-DYNAMICS STUDY OF MELTING AND FREEZING OF SMALL LENNARD-JONES CLUSTERS. Journal of Physical Chemistry 91, 4950-4963.

Ivanisenko, Y., Enikeev, N.A., Yang, K., Smoliakov, A., Soloviev, V.P., Fecht, H., Hahn, H., 2016. Contribution of grain boundary related strain accommodation to deformation of ultrafine-grained palladium. Materials Science and Engineering aStructural Materials Properties Microstructure and Processing 668, 255-262. Jang, S., Purohit, Y., Irving, D.L., Padgett, C., Brenner, D., Scattergood, R.O., 2008. Influence of $\mathrm{Pb}$ segregation on the deformation of nanocrystalline $\mathrm{Al}$ : Insights from molecular simulations. Acta Materialia 56, 4750-4761. Jennings, A.T., Weinberger, C.R., Lee, S.W., Aitken, Z.H., Meza, L., Greer, J.R., 2013. Modeling dislocation nucleation strengths in pristine metallic nanowires under experimental conditions. Acta Materialia 61, 2244-2259.

Khalajhedayati, A., Pan, Z.L., Rupert, T.J., 2016. Manipulating the interfacial structure of nanomaterials to achieve a unique combination of strength and ductility. Nature Communications 7. 
Kuhr, B., Farkas, D., Robertson, I.M., 2016. Atomistic studies of hydrogen effects on grain boundary structure and deformation response in FCC Ni. Computational Materials Science 122, 92-101.

Massalski, T.B., Okamoto, H., ASM International., 1990. Binary alloy phase diagrams, 2nd ed. ASM International, Materials Park, Ohio.

Millett, P.C., Selvam, R.P., Saxena, A., 2007. Stabilizing nanocrystalline materials with dopants. Acta Materialia 55, 2329-2336.

Min, J.Y., Hector, L.G., Lin, J.P., Carter, J.T., Sachdev, A.K., 2014. Spatio-temporal characteristics of propagative plastic instabilities in a rare earth containing magnesium alloy. International Journal of Plasticity 57, 52-76.

Mishin, Y., Farkas, D., 1998. Atomistic simulation of 001 symmetrical tilt grain boundaries in NiAl. Philosophical Magazine a-Physics of Condensed Matter Structure Defects and Mechanical Properties 78, 29-56.

Olmsted, D.L., Hector, L.G., Curtin, W.A., 2006. Molecular dynamics study of solute strengthening in $\mathrm{Al} / \mathrm{Mg}$ alloys. Journal of the Mechanics and Physics of Solids 54, 1763-1788.

Olmsted, D.L., Hector, L.G., Curtin, W.A., Clifton, R.J., 2005. Atomistic simulations of dislocation mobility in $\mathrm{Al}, \mathrm{Ni}$ and $\mathrm{Al} / \mathrm{Mg}$ alloys. Modelling and Simulation in Materials Science and Engineering 13, 371-388.

Ozerinc, S., Tai, K.P., Vo, N.Q., Bellon, P., Averback, R.S., King, W.P., 2012. Grain boundary doping strengthens nanocrystalline copper alloys. Scripta Materialia 67, 720-723.

Plimpton, S., 1995. FAST PARALLEL ALGORITHMS FOR SHORT-RANGE MOLECULAR-DYNAMICS. J. Comput. Phys. 117, 1-19.

Rajgarhia, R.K., Spearot, D.E., Saxena, A., 2010. Plastic deformation of nanocrystalline copper-antimony alloys. Journal of Materials Research 25, 411-421.

Randle, V., 2006. 'Special' boundaries and grain boundary plane engineering. Scripta Materialia 54, 1011-1015.

Rittner, J.D., Seidman, D.N., 1996. <110> symmetric tilt grain-boundary structures in fcc metals with low stacking-fault energies. Physical Review B 54, 6999-7015. Sadigh, B., Erhart, P., Stukowski, A., Caro, A., Martinez, E., Zepeda-Ruiz, L., 2012. Scalable parallel Monte Carlo algorithm for atomistic simulations of precipitation in alloys. Physical Review B 85.

Salehinia, I., Wang, J., Bahr, D.F., Zbib, H.M., 2014. Molecular dynamics simulations of plastic deformation in $\mathrm{Nb} / \mathrm{NbC}$ multilayers. International Journal of Plasticity 59 , 119-132.

Schaefer, J., Stukowski, A., Albe, K., 2011. Plastic deformation of nanocrystalline Pd$\mathrm{Au}$ alloys: On the interplay of grain boundary solute segregation, fault energies and grain size. Acta Materialia 59, 2957-2968.

Schafer, J., Stukowski, A., Albe, K., 2013. On the hierarchy of deformation processes in nanocrystalline alloys: Grain boundary mediated plasticity vs. dislocation slip. Journal of Applied Physics 114.

Spearot, D.E., 2008. Evolution of the E structural unit during uniaxial and constrained tensile deformation. Mechanics Research Communications 35, 81-88. Spearot, D.E., Tschopp, M.A., Jacob, K.I., McDowell, D.L., 2007. Tensile strength of < $100>$ and $<110>$ tilt bicrystal copper interfaces. Acta Materialia 55, 705-714. 
Stukowski, A., 2010. Visualization and analysis of atomistic simulation data with OVITO-the Open Visualization Tool. Modelling and Simulation in Materials Science and Engineering 18.

Stukowski, A., 2012. Structure identification methods for atomistic simulations of crystalline materials. Modelling and Simulation in Materials Science and Engineering 20.

Tschopp, M.A., Murdoch, H.A., Kecskes, L.J., Darling, K.A., 2014. "Bulk"

Nanocrystalline Metals: Review of the Current State of the Art and Future

Opportunities for Copper and Copper Alloys. Jom 66, 1000-1019.

Tschopp, M.A., Tucker, G.J., McDowell, D.L., 2007. Structure and free volume of $<110$ $>$ symmetric tilt grain boundaries with the E structural unit. Acta Materialia 55, 3959-3969.

Tucker, G.J., Tschopp, M.A., McDowell, D.L., 2010. Evolution of structure and free volume in symmetric tilt grain boundaries during dislocation nucleation. Acta Materialia 58, 6464-6473.

Upadhyay, M.V., Capolungo, L., Taupin, V., Fressengeas, C., Lebensohn, R.A., 2016. A higher order elasto-viscoplastic model using fast Fourier transforms: Effects of lattice curvatures on mechanical response of nanocrystalline metals. International Journal of Plasticity 83, 126-152.

Vo, N.Q., Schaefer, J., Averback, R.S., Albe, K., Ashkenazy, Y., Bellon, P., 2011. Reaching theoretical strengths in nanocrystalline $\mathrm{Cu}$ by grain boundary doping. Scripta Materialia 65, 660-663.

Williams, P.L., Mishin, Y., Hamilton, J.C., 2006. An embedded-atom potential for the $\mathrm{Cu}-\mathrm{Ag}$ system. Modelling and Simulation in Materials Science and Engineering 14, 817-833.

Yuan, R., Beyerlein, I.J., Zhou, C.Z., 2016. Statistical dislocation activation from grain boundaries and its role in the plastic anisotropy of nanotwinned copper. Acta Materialia 110, 8-18.

Zhang, L., Lu, C., Tieu, K., Pei, L.Q., Zhao, X., Cheng, K.Y., 2015. Molecular dynamics study on the grain boundary dislocation source in nanocrystalline copper under tensile loading. Materials Research Express 2.

Zhu, T., Li, J., Samanta, A., Leach, A., Gall, K., 2008. Temperature and strain-rate dependence of surface dislocation nucleation. Physical Review Letters 100. 
Figure 1. a) The bi-crystal simulation system; the MC swap regions are indicated by dashed lines. b) Structure and principal segregation sites at the $\Sigma 11(332)$ [110] STGB. The GB sites are marked following the notations introduced in (Duparc et al., 2005). The arrows show the displacement of atomic planes during leading partial dislocation emission. The view is along the [110] tilt axis, with the open and filled circles representing the atoms on two different $\langle 110\rangle$ planes. c) The system with one emitted dislocation used in the energy calculations (Eqs. 1-3). All solutes were located at the bottom GB. d) A typical case of the simultaneous nucleation of multiple dislocations (fish-bone structure) observed in the MD simulations of tensile loading (the direction of the applied deformation is indicated by the arrows). The atoms in a), c) and d) are colored according to the Common Neighbor Analysis (CNA) (Honeycutt and Andersen, 1987; Stukowski, 2012) and fcc (perfect bulk) atoms are not shown. The color-coding is as follows: red - hcp, grey - other. The red features in c) and d) are the intrinsic stacking faults trailing leading partial dislocations. Analysis and visualization of MD simulation snapshots were performed using the software package OVITO (Stukowski, 2010).

Figure 2. Change in the total energy during preparation of the initial model of $\mathrm{Cu}$, with the largest $\mathrm{Ag}$ concentration considered in the present study. To compare the energy during $\mathrm{MC}$ simulation on the rigid lattice with the energies calculated during MD simulations, the value of $3 \mathrm{Nk}_{\mathrm{B}} \mathrm{T}$ was added to the former.

Figure 3. Stress-strain curves obtained using different solute concentrations.

Figure 4. Yield stress vs. total solute concentration in the simulation cell.

Figure 5. The solute distribution used when the solute atoms occupy every third $\mathrm{A}^{\prime}$ segregation site.

Figure 6. Yield stresses for different segregation sites. a) $\Delta \mathrm{E}_{1}$ corresponds to the case of a single solute atom, and the energy minimization performed at zero strain. b) same as (a) but $\Delta \mathrm{E}_{3}$ corresponds to the case of solute atoms placed at every third segregation site. $\mathrm{c}$ ) the same as (b) but with the energy minimization performed at $1 \%$ strain. 

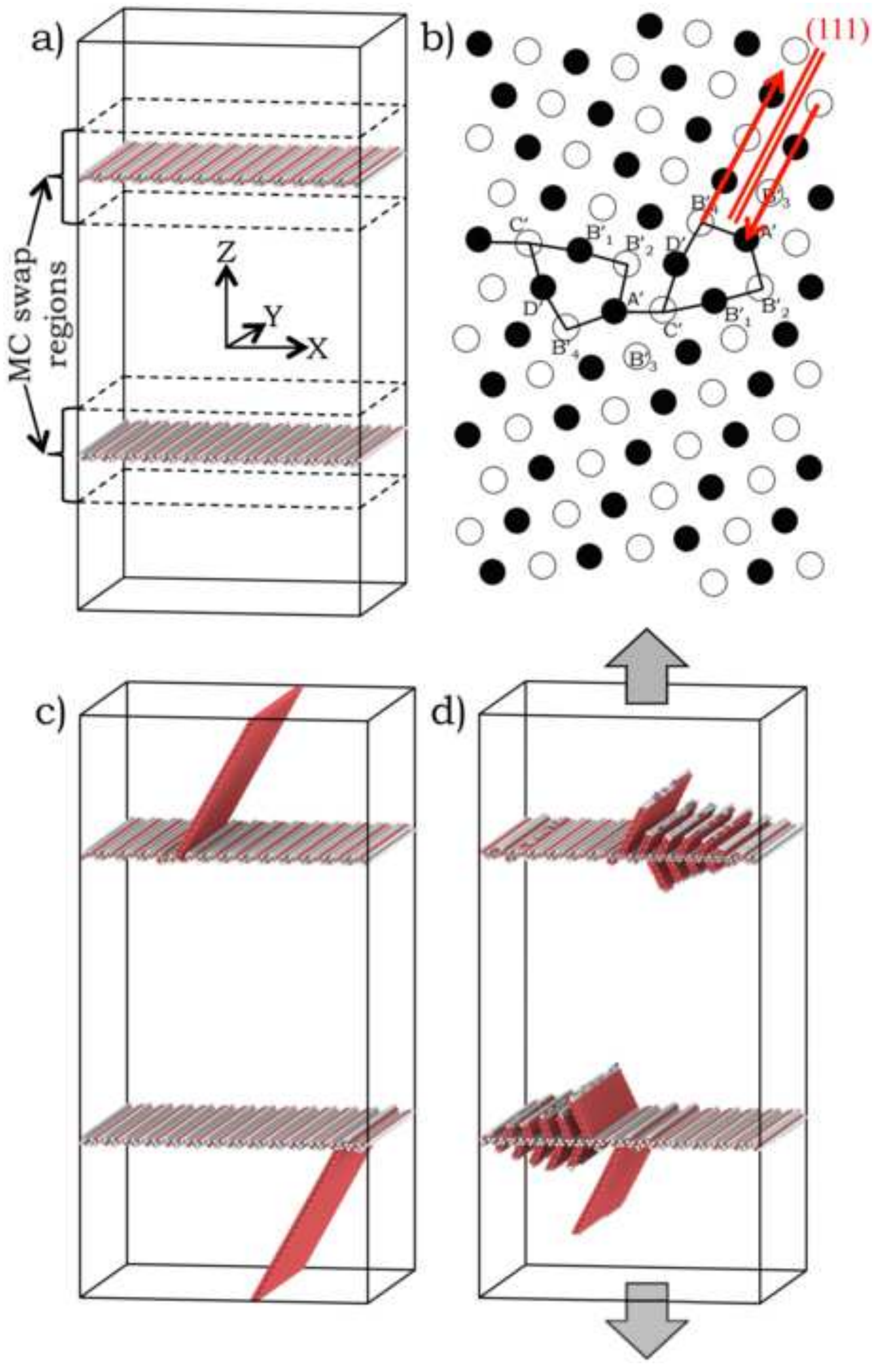

Figure 1 


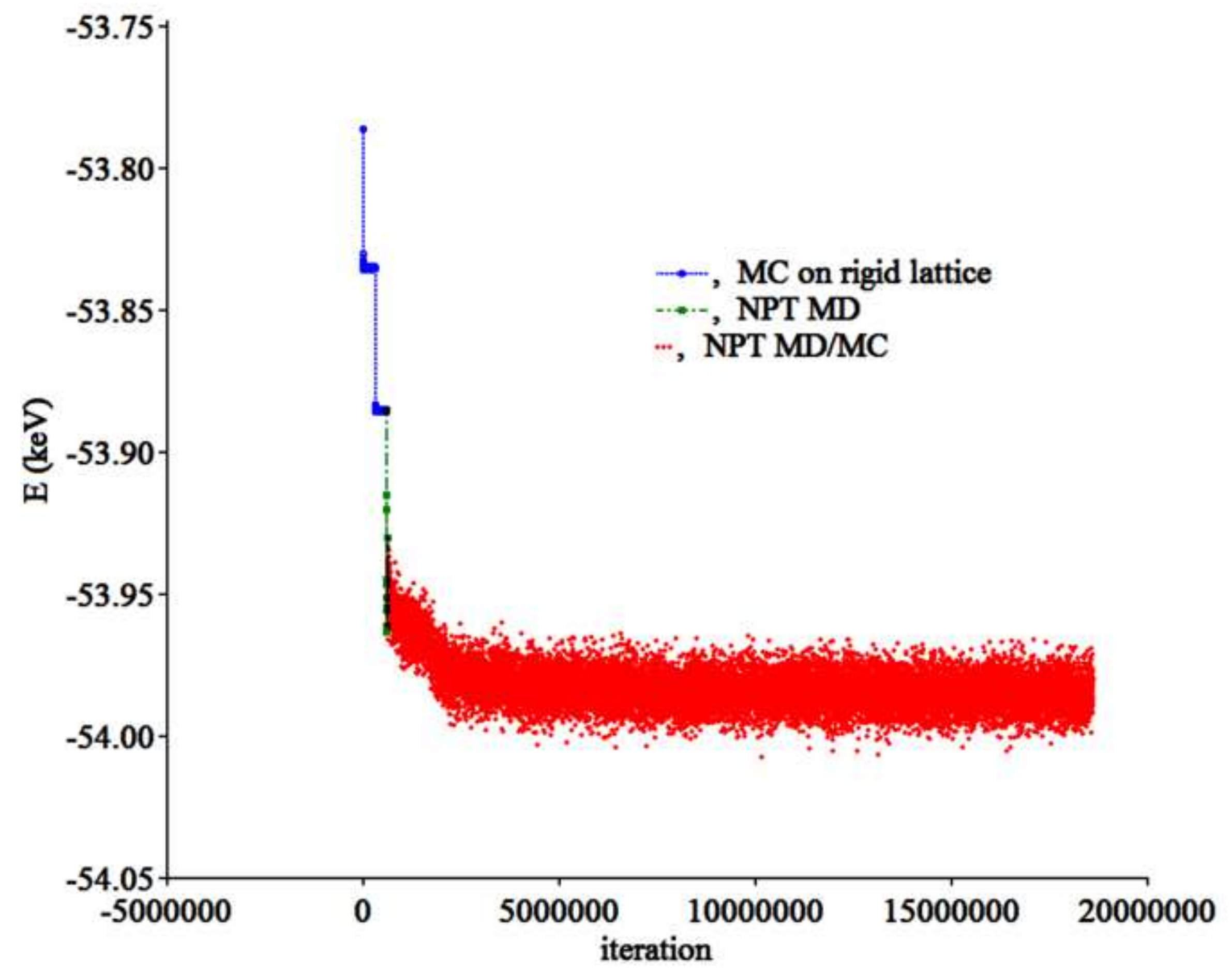



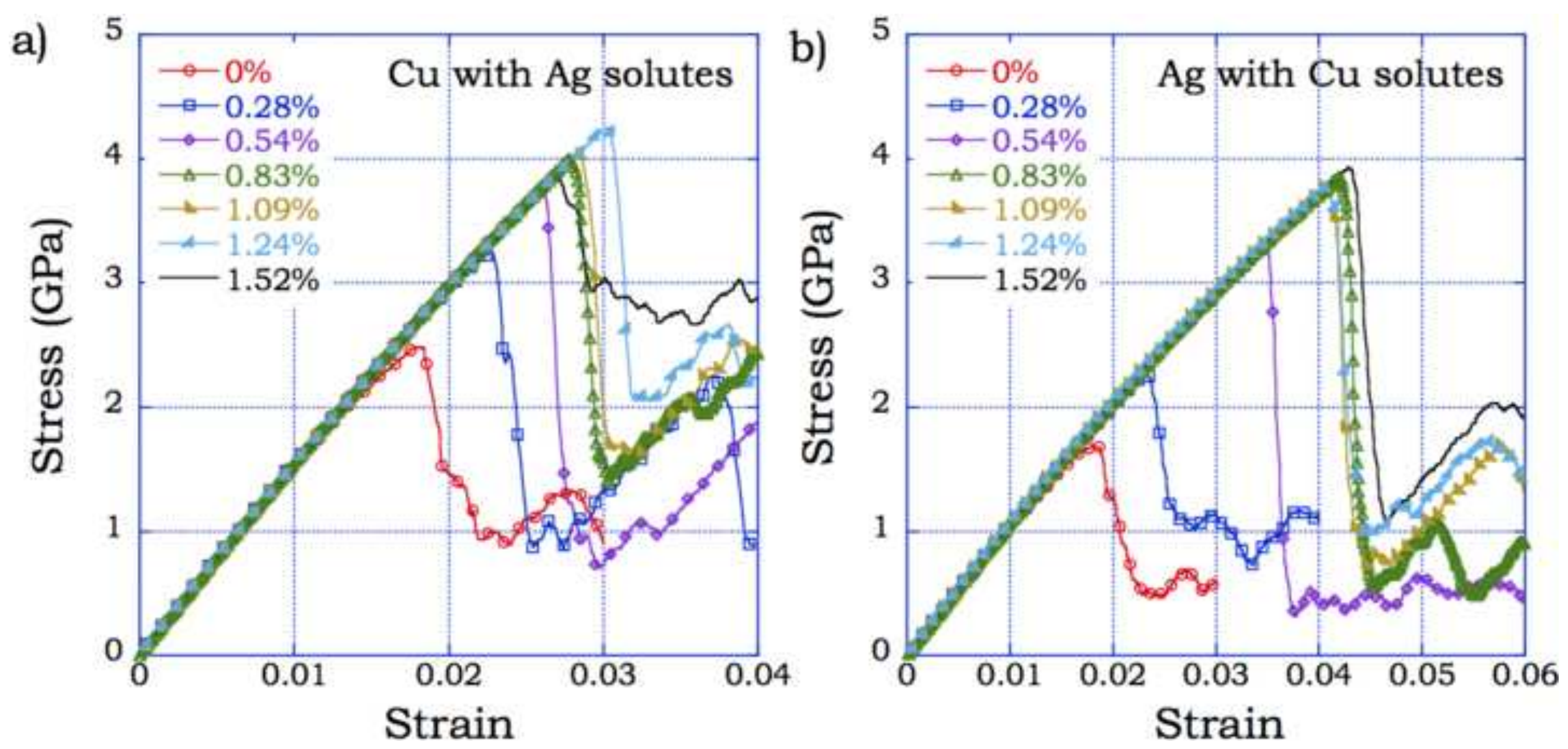


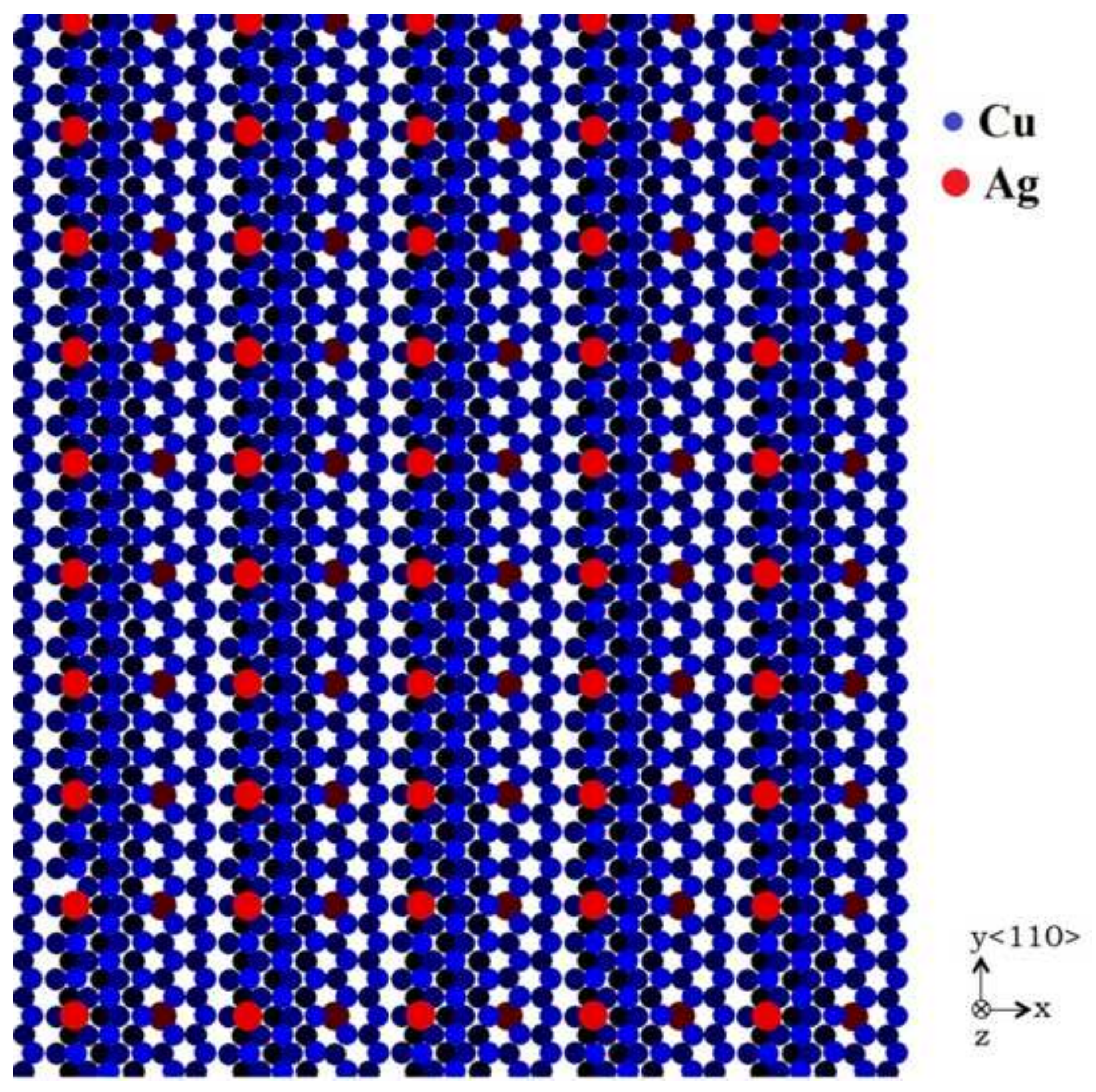



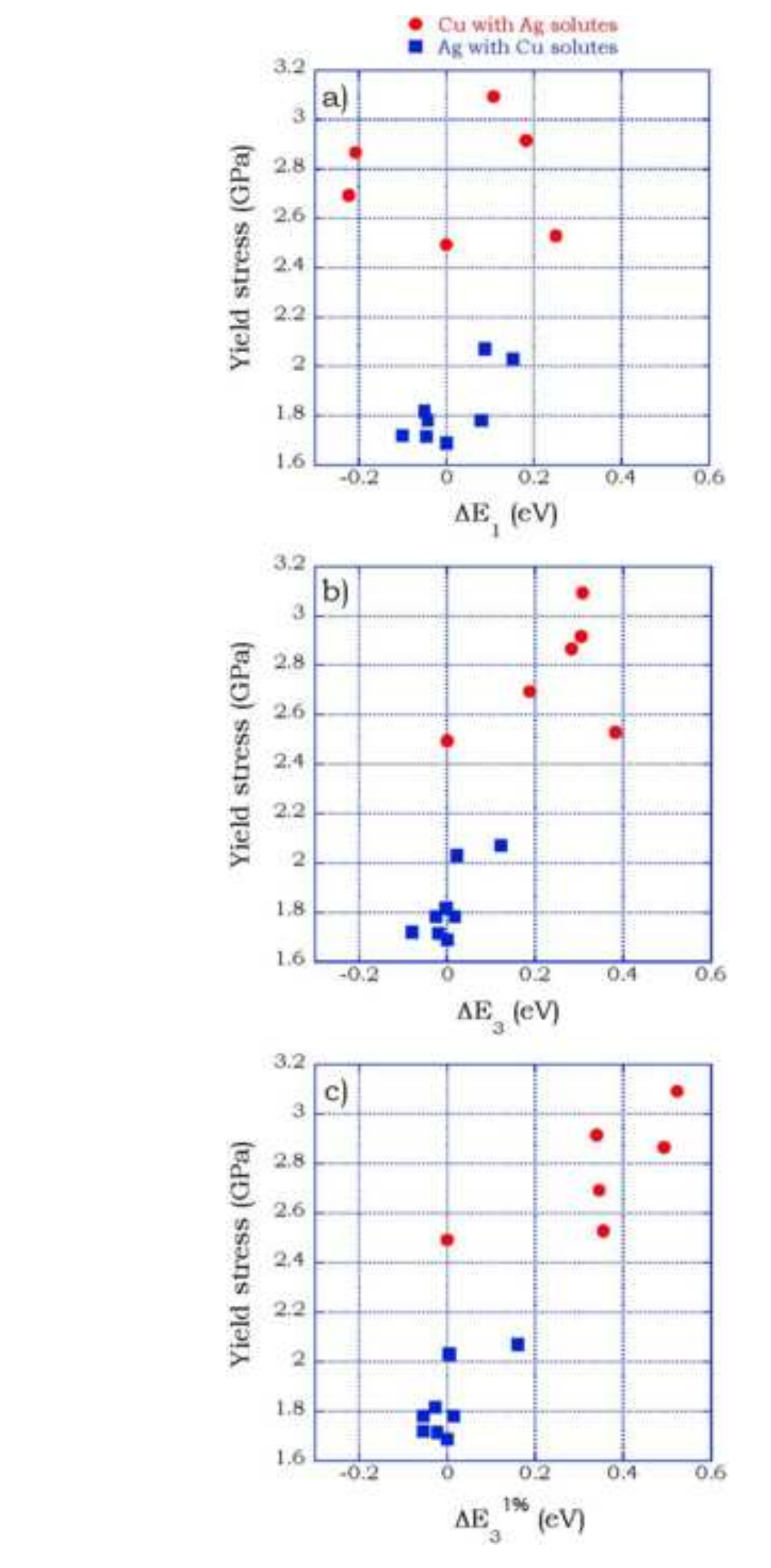

Figure 6

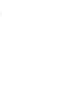

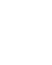

\section{Figure 6}

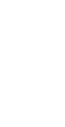

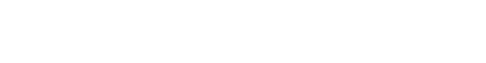

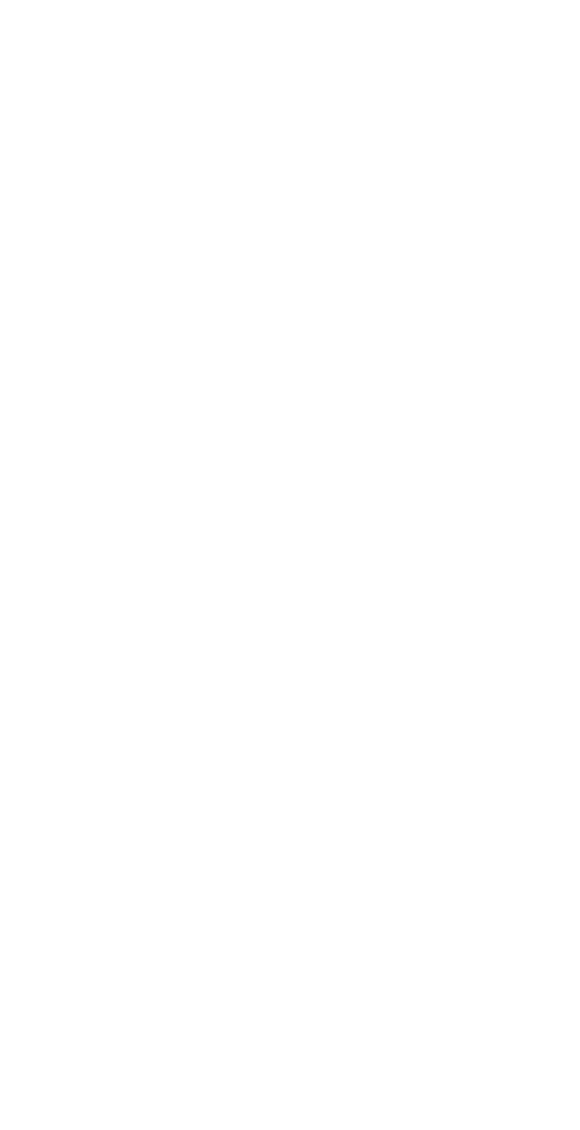


Table I. Heat of segregation and effect of solutes on the change in energy associated with the dislocation emission. All energies are expressed in eV/atom. The values for the most favorable segregation sites are shown in bold. The values of $\Delta E_{3}$ are shown in italic if the GB experiences a reconstruction during the tensile loading simulation.

\begin{tabular}{|c|c|c|c|c|c|c|c|c|}
\hline \multirow{2}{*}{ Site } & \multicolumn{4}{|c|}{$\mathrm{Ag}$ in $\mathrm{Cu}$} & \multicolumn{4}{c|}{$\mathrm{Cu}$ in $\mathrm{Ag}$} \\
\cline { 2 - 9 } & $E_{\text {seg }}$ & $\Delta E_{1}$ & $\Delta E_{3}$ & $\Delta E_{3}^{1 \%}$ & $E_{\text {seg }}$ & $\Delta E_{1}$ & $\Delta E_{3}$ & $\Delta E_{3}^{1 \%}$ \\
\hline $\mathrm{A}^{\prime}$ & $\mathbf{- 0 . 4 2 0}$ & $\mathbf{0 . 1 8 2}$ & $\mathbf{0 . 3 0 6}$ & $\mathbf{0 . 3 3 9}$ & 0.130 & -0.045 & -0.019 & -0.023 \\
\hline $\mathrm{B}_{1}^{\prime}$ & -0.210 & 0.205 & 0.090 & 0.118 & -0.050 & -0.042 & 0.017 & 0.015 \\
\hline $\mathrm{B}_{2}^{\prime}$ & -0.120 & 0.250 & 0.382 & 0.355 & 0.012 & -0.099 & -0.080 & -0.054 \\
\hline $\mathrm{B}_{3}^{\prime}$ & -0.170 & 0.107 & 0.308 & 0.523 & 0.050 & -0.049 & -0.004 & -0.029 \\
\hline $\mathrm{B}_{4}^{\prime}$ & -0.003 & -0.076 & -0.228 & -0.282 & -0.080 & 0.088 & 0.124 & 0.159 \\
\hline $\mathrm{C}^{\prime}$ & -0.010 & -0.208 & 0.284 & 0.492 & -0.015 & 0.081 & -0.025 & -0.055 \\
\hline $\mathrm{D}^{\prime}$ & 0.110 & -0.222 & 0.187 & 0.346 & $\mathbf{- 0 . 2 0 0}$ & $\mathbf{0 . 1 5 4}$ & $\mathbf{0 . 0 2 2}$ & $\mathbf{0 . 0 0 6}$ \\
\hline
\end{tabular}

